\title{
Tracing the redshift evolution of Hubble parameter with gravitational-wave standard sirens
}

$\operatorname{AUTHOR}(S)$ :

Nishizawa, Atsushi; Taruya, Atsushi; Saito, Shun

\section{CITATION:}

Nishizawa, Atsushi ... [et al]. Tracing the redshift evolution of Hubble parameter with gravitational-wave standard sirens. Physical Review D 2011, 83(8): 084045.

ISSUE DATE:

2011-04

URL:

http://hdl.handle.net/2433/145982

RIGHT:

(C) 2011 American Physical Society 
PHYSICAL REVIEW D 83, 084045 (2011)

\title{
Tracing the redshift evolution of Hubble parameter with gravitational-wave standard sirens
}

\author{
Atsushi Nishizawa, ${ }^{1, *}$ Atsushi Taruya, ${ }^{2,3}$ and Shun Saito ${ }^{4,5}$ \\ ${ }^{1}$ Yukawa Institute for Theoretical Physics, Kyoto University, Kyoto 606-8502, Japan \\ ${ }^{2}$ Research Center for the Early Universe, Graduate School of Science, The University of Tokyo, Tokyo 113-0033, Japan \\ ${ }^{3}$ Institute for the Physics and Mathematics of the Universe, The University of Tokyo, Kashiwa, Chiba 277-8568, Japan \\ ${ }^{4}$ Department of Physics, Graduate School of Science, The University of Tokyo, Tokyo 113-0033, Japan \\ ${ }^{5}$ Department of Astronomy, 601 Campbell Hall, University of California, Berkeley, California 94720, USA
}

(Received 19 December 2010; published 26 April 2011)

\begin{abstract}
Proposed space-based gravitational-wave detectors such as BBO and DECIGO can detect $\sim 10^{6}$ neutron star (NS) binaries and determine the luminosity distance to the binaries with high precision. Combining the luminosity distance and electromagnetically derived redshift, one would be able to probe cosmological expansion out to high redshift. In this paper, we show that the Hubble parameter as a function of redshift can be directly measured with monopole and dipole components of the luminosity distance on the sky. As a result, the measurement accuracies of the Hubble parameter in each redshift bin up to $z=1$ are 3-14\%, $1.5-8 \%$, and $0.8-4 \%$ for the observation time $1 \mathrm{yr}, 3 \mathrm{yr}$, and $10 \mathrm{yr}$, respectively.
\end{abstract}

DOI: 10.1103/PhysRevD.83.084045

PACS numbers: 04.30. $-\mathrm{w}, 95.36 .+\mathrm{x}, 98.80 .-\mathrm{k}$

\section{INTRODUCTION}

Future space-based gravitational-wave detectors such as DECI-hertz Interferometer Gravitational-wave Observatory (DECIGO) [1,2] and Big-Bang Observer (BBO) [3] (see also [4] for updated information) are the most sensitive to a gravitational wave $(\mathrm{GW})$ in $0.1-1 \mathrm{~Hz}$ band and will aim at detecting a cosmological GW background generated during the inflationary epoch, the mergers of intermediate-mass black holes, and a large number of neutron star (NS) binaries in an inspiraling phase. These GW sources enable us to measure the cosmological expansion with unprecedented precision [4], to investigate the population and formation history of compact-binary objects, and to test alternative theories of gravity $[5,6]$.

It has been known that the continuous GW signal from a compact-binary object provides a unique way to measure the luminosity distance to the source with high precision. Such binary sources are often referred to as standard siren (analogous to the electromagnetic standard candle). With the redshift information determined by an electromagnetic follow-up observation, the standard siren can be an accurate tracer of the cosmic expansion [7]. The feasibility of the standard siren relies on the determination of the redshift of each binary. The identification of a host galaxy by follow-up observation is thus crucial, indicating that a high-angular resolution is generally required for GW detector. In the case of DECIGO and BBO, the detectors orbit the Sun with a period of one sidereal year, and constitute four clusters, each of which consists of three spacecrafts exchanging laser beams with the others. The two of the four clusters are located at the same position to enhance correlation sensitivity to a gravitational-wave background, and the other two are separated on the Earth orbit in order

\footnotetext{
*anishi@yukawa.kyoto-u.ac.jp
}

to enhance the angular resolution so that we can easily identify the host galaxy of each NS binary via the electromagnetic follow-up observations. Based on this setup, Cutler and Holz [4] have shown that cosmological parameters can be accurately measured by DECIGO and BBO with a precision of $\sim 1 \%$, assuming a large number of neutron star (NS) binaries, $\sim 10^{6}$.

In this paper, we show that the space-based GW detectors can also measure the Hubble parameter $H(z)$ from the GW standard sirens [8]. In this method, the measured quantity is independent of that in the usual method of a standard siren, in which an observable is the luminosity distance as an integrated quantity of $H^{-1}(z)$. The method to measure the Hubble parameter at each redshift has been proposed by Bonvin et al. [9,10], who originally developed this idea in the observation of distant type Ia supernovae. In general, a large number of samples is necessary for the accurate measurement of $H(z)$, and they concluded that $10^{5}-10^{6}$ supernovae are needed to achieve a few percent accuracy. In contrast to the supernovae observation which requires an unrealistically large number of the samples and suffers from relatively large systematics, DECIGO and BBO are expected to detect a million NS binaries with smaller systematic errors. Thus, the measurement of the Hubble parameter at high redshifts becomes even more feasible with standard sirens.

The Hubble parameter $H(z)$ can be also measured by estimating the differential age of the oldest galaxies in each redshift bin [11] and using the baryon acoustic oscillation (BAO) along the line-of-sight direction from the spectroscopic galaxy samples (e.g., $[12,13])$. In this respect, the present method with GW observation is complementary, and useful for an independent cross-check.

This paper is organized as follows. In Sec. II, we briefly review the basic idea to measure the Hubble parameter via dipole of the luminosity distance $d_{L}$, originally proposed 
by Bonvin et al. $[9,10]$, and derive the basic equations to estimate the accuracy of the Hubble parameter. In Sec. III, the method is applied to the GW observation. We briefly describe the GW standard siren and calculate the measurement accuracy of the Hubble parameter. In Sec. IV, systematic errrors are discussed and compared with the uncertainty coming from instrumental noise in GW observation. Finally, Sec. V gives a brief summary and discussions on the feasibility of our method. Throughout the paper, we adopt units $c=G=1$, and assume a flat Universe.

\section{HUBBLE PARAMETER FROM THE DIPOLE ANISOTROPY OF LUMINOSITY DISTANCE}

Consider the luminosity distance to some astronomical objects measured at redshift $z$ and angular position $\mathbf{n}$. In principle, the observations of many objects over the sky enables us to map out the angular distribution of luminosity distance, $d_{L}(z, \mathbf{n})$, and no directional dependence appears if the observer is at cosmological rest-frame (i.e., CMB rest-frame) in a homogeneous universe. However, there certainly exist tiny anisotropies in $d_{L}$ arising from the matter inhomogeneities of the large-scale structure and/or the local motion of the observer [14]. As it has been shown in Ref. [10], the dominant component of anisotropies is the dipole induced by the peculiar velocity of the observer, and the contribution to the higher multipoles coming from the weak gravitational lensing effect is basically small [15]. Then, we can expand the luminosity distance as

$$
\begin{aligned}
& d_{L}(z, \mathbf{n})=d_{L}^{(0)}(z)+d_{L}^{(1)}(z) \cos \theta ; \\
& d_{L}^{(0)}(z) \equiv \frac{1}{4 \pi} \int d \mathbf{n} d_{L}(z, \mathbf{n}), \\
& d_{L}^{(1)}(z) \equiv \frac{3}{4 \pi} \int d \mathbf{n}(\mathbf{n} \cdot \mathbf{e}) d_{L}(z, \mathbf{n}),
\end{aligned}
$$

where we define $\cos \theta=\mathbf{n} \cdot \mathbf{e}$, and the quantity $\mathbf{e}$ is the unit vector directed toward the dipole.

In the expression (1), the first term in the right-hand side is the direction-averaged luminosity distance, which is identified with the one defined in the homogeneous and isotropic universe:

$$
d_{L}^{(0)}(z)=(1+z) \int_{0}^{z} \frac{d z^{\prime}}{H\left(z^{\prime}\right)} ;
$$

with the Hubble parameter given by

$$
\begin{aligned}
H(z)= & H_{0}\left\{\Omega_{m}(1+z)^{3}+\left(1-\Omega_{m}\right)(1+z)^{3\left(1+w_{0}+w_{a}\right)}\right. \\
& \left.\times \exp \left[-3 w_{a} \frac{z}{1+z}\right]\right\}^{1 / 2} .
\end{aligned}
$$

Here we assumed the flat Universe, and the dark energy equation-of-state parameter $w(a)=P / \rho$ parametrized by $w(a)=w_{0}+w_{a}(1-a)$. For later analysis of the error estimation, we adopt a fiducial set of cosmological parameters: $\Omega_{m}=0.3, \quad w_{0}=-1, \quad w_{a}=0, \quad$ and $H_{0}=$ $72 \mathrm{~km} \mathrm{~s}^{-1} \mathrm{Mpc}^{-1}$.

On the other hand, the second term on the right-handside of Eq. (1) arises from the Doppler effect due to the motion of the observer. To derive the expression for $d_{L}^{(1)}$, we approximate the propagation of $\mathrm{GW}$ or light following the trajectory of a null geodesic. Writing the luminosity distance to an astronomical object as a function of the conformal time $\eta$ at which the source emits GW or light, the Doppler effect leads to $[9,10]$

$$
d_{L}(\eta, \mathbf{n})=d_{L}^{(0)}(\eta)\left[1-\mathbf{n} \cdot \mathbf{v}_{0}\right]
$$

where the vector $\mathbf{v}_{0}$ indicates the peculiar velocity of a local observer, which is small enough relative to the light velocity. Note that the motion of the local observer also induces the Doppler effect in the redshift, $z=\bar{z}+\delta z$, where the unperturbed redshift $\bar{z}$ is defined as $\bar{z}=$ $a^{-1}(\eta)-1$. To first order in $\mathbf{v}_{0}$, we have

$$
\delta z=-\left(\mathbf{n} \cdot \mathbf{v}_{0}\right)(1+\bar{z}) .
$$

Rewriting Eq. (5) in terms of the redshift $z$, then expanding $d_{L}^{(0)}(z-\delta z)$ up to the first order, and using Eqs. (3) and (6) give

$$
\begin{aligned}
d_{L}(z, \mathbf{n}) & =\left[d_{L}^{(0)}(z)-\frac{\partial d_{L}^{(0)}(z)}{\partial z} \delta z\right]\left[1-\mathbf{n} \cdot \mathbf{v}_{0}\right] \\
& =d_{L}^{(0)}(z)+\frac{(1+z)^{2}}{H(z)}\left(\mathbf{n} \cdot \mathbf{v}_{0}\right)
\end{aligned}
$$

Comparing the above expression with Eqs. (1) and (2), we arrive at

$$
d_{L}^{(1)}(z)=\frac{\left|\mathbf{v}_{0}\right|(1+z)^{2}}{H(z)}
$$

with the direction of the dipole specifically chosen as $\mathbf{e}=$ $\mathbf{v}_{0} /\left|\mathbf{v}_{0}\right|$. In the expression (8), the magnitude of dipole anisotropy is inversely proportional to the Hubble parameter at the source redshift, because the perturbed luminosity distance corresponds to the derivative of Eq. (3). Recalling the fact that the motion of the local observer also induces the same size of dipole anisotropy in the CMB and its amplitude is estimated as $\left|\mathbf{v}_{0}\right|=369.1 \pm 0.9 \mathrm{~km} \mathrm{~s}^{-1}$ [16]. Then the dipole anisotropy in the luminosity distance to high- $z$ objects gives a direct measure of $H(z)$.

Now, let us discuss the statistical error in the measurement of the Hubble parameter. We add the measurement error of the luminosity distance $\delta d_{L}(z, \mathbf{n})$ into Eq. (1):

$$
d_{L}(z, \mathbf{n})=d_{L}^{(0)}(z)+d_{L}^{(1)}(z)(\mathbf{n} \cdot \mathbf{e})+\delta d_{L}(z, \mathbf{n}) .
$$

Then the measurement error of $d_{L}^{(1)}$ is estimated from the definition of $d_{L}^{(1)}$, and is expressed as 
TRACING THE REDSHIFT EVOLUTION OF HUBBLE ..

$$
\delta d_{L}^{(1)}(z)=\frac{3}{4 \pi} \int d \mathbf{n}(\mathbf{e} \cdot \mathbf{n}) \delta d_{L}(z, \mathbf{n}) .
$$

The variance of this becomes

$$
\begin{aligned}
{\left[\Delta d_{L}^{(1)}(z)\right]^{2} \equiv } & \left\langle\left[\delta d_{L}^{(1)}(z)\right]^{2}\right\rangle \\
= & \left(\frac{3}{4 \pi}\right)^{2} \int d \mathbf{n} \int d \mathbf{n}^{\prime} \times(\mathbf{e} \cdot \mathbf{n})\left(\mathbf{e} \cdot \mathbf{n}^{\prime}\right) \\
& \times\left\langle\delta d_{L}(z, \mathbf{n}) \delta d_{L}\left(z, \mathbf{n}^{\prime}\right)\right\rangle,
\end{aligned}
$$

where the bracket represents the ensemble average over the sources given at $z$. Assuming that the distance errors measured from each GW source are statistically independent and isotropic, we have

$$
\left\langle\delta d_{L}(z, \mathbf{n}) \delta d_{L}\left(z, \mathbf{n}^{\prime}\right)\right\rangle=4 \pi\left[\Delta d_{L}^{(0)}(z)\right]^{2} \delta^{2}\left(\mathbf{n}-\mathbf{n}^{\prime}\right)
$$

where the quantity $\left[\Delta d_{L}^{(0)}(z)\right]^{2}$ is the variance of $d_{L}^{(0)}$. Substituting this into Eq. (9), we obtain

$$
\left[\Delta d_{L}^{(1)}(z)\right]^{2}=\frac{9}{4 \pi}\left[\Delta d_{L}^{(0)}(z)\right]^{2} \int d \mathbf{n}(\mathbf{e} \cdot \mathbf{n})^{2},=3\left[\Delta d_{L}^{(0)}(z)\right]^{2} .
$$

From this and the expression (8), the measurement error of the Hubble parameter at a given redshift $z$ is related to the error of the direction-averaged luminosity distance, $\Delta d_{L}^{(0)}(z)$, and is given by

$$
\frac{\Delta H(z)}{H(z)}=\frac{\Delta d_{L}^{(1)}(z)}{d_{L}^{(1)}(z)}=\sqrt{3}\left[\frac{d_{L}^{(1)}(z)}{d_{L}^{(0)}(z)}\right]^{-1}\left[\frac{\Delta d_{L}^{(0)}(z)}{d_{L}^{(0)}(z)}\right] .
$$

Here we ignored the velocity error in CMB observation. In fact, the contribution is negligible, as we will show in Sec. IV. The factor $\left[d_{L}^{(1)} / d_{L}^{(0)}\right]^{-1}$ is typically very large in the present case with $\left|\mathbf{v}_{0}\right| \ll 1$. In Fig. 1, we plot the ratio

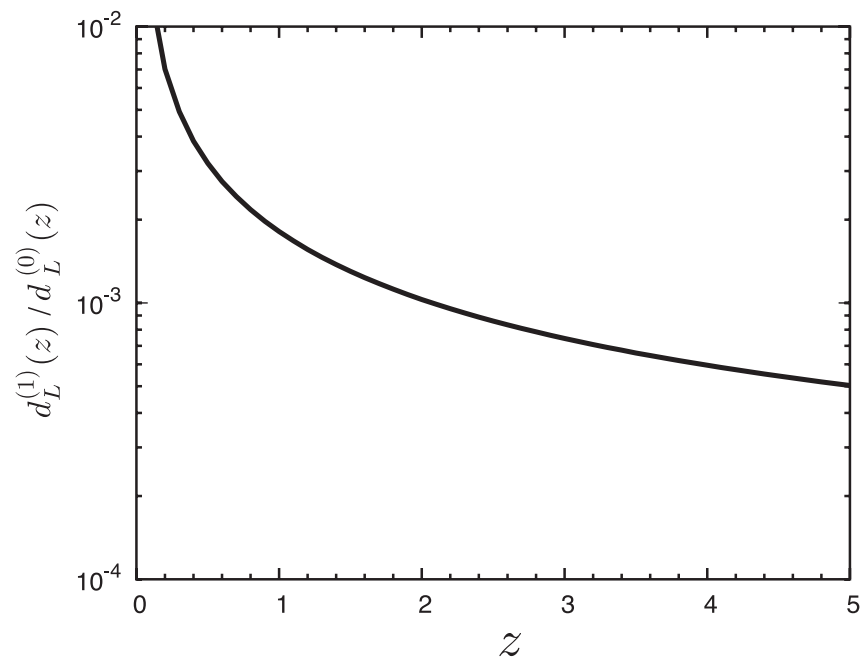

FIG. 1. Ratio of $d_{L}^{(1)}(z)$ to $d_{L}^{(0)}(z)$, in which the CMB dipole of $\left|v_{0}\right|=369.1 \pm 0.9 \mathrm{~km} / \mathrm{sec}$ measured by Wilkinson Microwave Anisotropy Probe (WMAP) [16] is used. of the dipole to the monopole in the luminosity distance. The figure implies that even a negligibly small error in the averaged distance $d_{L}^{(0)}$ can produce a large scatter in $H(z)$.

Note that the mean error on the Hubble parameter is reduced to $\Delta H / \sqrt{N}$ if we observe $N$ independent sources at a given redshift bin. Thus, for a precision measurement of Hubble parameter, we need not only an accurate determination of averaged distance to each source, $\Delta d_{L}^{(0)} / d_{L}^{(0)} \ll 1$ but also a large number of sources. In the case of the type Ia supernovae as standard candles, the averaged distance error is related to the intrinsic magnitude error $\Delta m$ as $\Delta d_{L}^{(0)} / d_{L}^{(0)}=(\ln 10 / 5) \Delta m$. Adopting an optimistic value $\Delta m=0.1$, it yields the error $\Delta d_{L}^{(0)} / d_{L}^{(0)} \approx$ 0.05 , but to achieve a few percent accuracy in the Hubble parameter, we need an unrealistically large number of samples of the order $10^{6}$. In other words, for a reasonable number of $10^{4}$ samples (see, e.g., $[17,18]$ ), the systematics in the averaged distance should be reduced to $\Delta m<0.01$, which seems very difficult from the empirical calibration.

\section{GW STANDARD SIRENS}

In this section, we consider the GW standard sirens as an alternative probe to measure the Hubble parameter from the dipole anisotropy of luminosity distance. The advantage of using the standard sirens observed by space-based GW detectors is that the expected number of sources (NS binaries) is much larger than that of the type Ia supernovae, and the sources are distributed deeply enough at higher redshifts. Moreover, the NS binary would be a clean GW source, and with DECIGO or BBO, the luminosity distance $d_{L}(z, \mathbf{n})$ can be accurately measured with less systematics.

\section{A. Luminosity distance error}

Let us first estimate the distance error of the standard siren, taking account of the instrumental noise of the GW detector. Possible systematic errors will be discussed later. In GW experiments, a direct observable is the waveform of the GW signal, and comparing it with a theoretical template, we not only determine the system parameters of GW source but also extract the cosmological information.

For a single binary system, the Fourier transform of the GW waveform is expressed as a function of frequency $f[19,20]$,

$$
\tilde{h}(f)=\frac{A}{d_{L}(z)} M_{z}^{5 / 6} f^{-7 / 6} e^{i \Psi(f),}
$$

where $d_{L}$ is the luminosity distance, and the quantity $M_{z}=$ $(1+z) \eta^{3 / 5} M_{t}$ is the redshifted chirp mass with the total mass $M_{t}=m_{1}+m_{2}$ and the symmetric mass ratio $\eta=$ $m_{1} m_{2} / M_{t}^{2}$. Here, the constant $A$ is given by $A=$ $\left(\sqrt{6} \pi^{2 / 3}\right)^{-1}$, which is multiplied by the factor $\sqrt{4 / 5}$ for a geometrical average over the inclination angle of a binary [21]. The function $\Psi(f)$ represents the frequency- 
dependent phase arising from the orbital evolution, and at the order of the restricted 1.5 post-Newtonian $(\mathrm{PN})$ approximation, it is given by $[19,20]$

$$
\begin{aligned}
\Psi(f)= & 2 \pi f t_{c}-\phi_{c}-\frac{\pi}{4}+\frac{3}{128}\left(\pi M_{z} f\right)^{-5 / 3} \\
& \times\left[1+\frac{20}{9}\left(\frac{743}{336}+\frac{11}{4} \eta\right) \eta^{-2 / 5}\left(\pi M_{z} f\right)^{2 / 3}\right. \\
& \left.-16 \pi \eta^{-3 / 5}\left(\pi M_{z} f\right)\right],
\end{aligned}
$$

where $t_{c}$ and $\phi_{c}$ are the time and phase at coalescence, respectively. The first term in the bracket in Eq. (13) corresponds to Newtonian-order dynamics and the other remaining terms represent the post-Newtonian-order corrections in powers of $v \sim\left(\pi M_{z} f\right)^{1 / 3}$. In principle, there additionally appears a phase correction due to cosmic expansion, and the Hubble parameter $H(z)$ can be also measured from this term [1,22]. Although the inclusion of the phase correction slightly changes the size of the errors in binary parameters, it does not seriously affect the estimation of the luminosity distance $d_{L}$. In addition, the sensitivity of the phase correction to the Hubble parameter is rather small. Thus, we may safely ignore the phase correction due to cosmic expansion in the subsequent analysis.

In Eqs. (12) and (13), there are five unknown parameters to be determined observationally, i.e., $M_{z}, \eta, t_{c}, \phi_{c}$, and $d_{L}$. Except for the luminosity distance, the four parameters merely carry the information on the individual property of the binary system. For simplicity, we consider the equalmass NS binaries with $1.4 M_{\odot}$, which lead to $M_{z}=$ $1.22(1+z) M_{\odot}$ and $\eta=1 / 4$, and set the other parameters to $t_{c}=0$ and $\phi_{c}=0$.

Since the GW observation can only determine the redshifted chirp mass $M_{z}$, the redshift of each binary has to be measured from an electromagnetic counterpart. According to Cutler and Holz [4], the angular resolution of BBO is $\sim 1-100 \operatorname{arcsec}^{2}$, with which we can identify the host galaxy of the binary. We thus suppose that the redshift of any binary system is obtained from the electromagnetic observations. Note that the Doppler effect by the local motion also affects the redshifted chirp mass, and the dipole anisotropy might be measured through the spatial distribution of the observed chirp mass if the intrinsic scatter in the mass distribution of NS binaries is very small. The feasibility to measure the dipole anisotropy from the chirp mass might be interesting, but we need a more detailed study on the formation history of NS binaries, and we here simply ignore this effect in the parameter estimation.

The fundamental basis to estimate the distance error for a single binary is the Fisher matrix formalism. The Fisher matrix for a single binary is given by $[19,23]$

$$
\Gamma_{a b}=4 \sum_{i=1}^{8} \operatorname{Re} \int_{f_{\min }}^{f_{\max }} \frac{\partial_{a} \tilde{h}_{(i)}^{*}(f) \partial_{b} \tilde{h}_{(i)}(f)}{P(f)} d f,
$$

where $\partial_{a}$ denotes a derivative with respect to a parameter $\theta_{a} ; M_{z}, \eta, t_{c}, \phi_{c}$, and $d_{L}$. The quantity $\tilde{h}_{(i)}$ represents the $\mathrm{GW}$ signal obtained from the $i$-th interferometer. Since two independent signals are obtained for each cluster [24], DECIGO has the eight interferometric signals in total, each of which is supposed to have an identical detector response and noise power spectrum $P(f)$. The analytical fit of noise spectrum [25] is given by

$$
\begin{aligned}
P(f)= & 4.21 \times 10^{-50}\left(\frac{f}{1 \mathrm{~Hz}}\right)^{-4}+1.25 \times 10^{-47} \\
& +3.92 \times 10^{-49}\left(\frac{f}{1 \mathrm{~Hz}}\right)^{2} \mathrm{~Hz}^{-1} .
\end{aligned}
$$

In Fig. 2, the noise spectrum of DECIGO is shown, together with the evolutionary tracks of the NS binary located at three different redshifts, $z=0.1,1$, and 5 . In each track, the symbols indicate the frequency at the 10, 3 , and $1 \mathrm{yr}$ before the time of binary coalescence (from left to right). In this respect, the lower cutoff of the frequency $f_{\text {min }}$ should be incorporated into the integration in Eq. (14), and is given by the function of observation time $T_{\mathrm{obs}}$ as well as the redshift and mass:

$$
f_{\min }=0.233\left(\frac{1 M_{\odot}}{M_{z}}\right)^{5 / 8}\left(\frac{1 \mathrm{yr}}{T_{\mathrm{obs}}}\right)^{3 / 8} \mathrm{~Hz} .
$$

Note that the coalescence frequency of the NS binary is typically $\sim \mathrm{kHz}$, and thus the upper cutoff of the frequency naturally arises from the noise curve. For the computational purpose, we set $f_{\max }=100 \mathrm{~Hz}$.

Given the numerically evaluated Fisher matrix, the marginalized 1-sigma error of a parameter, $\Delta \theta_{a}$, is estimated from the inverse Fisher matrix

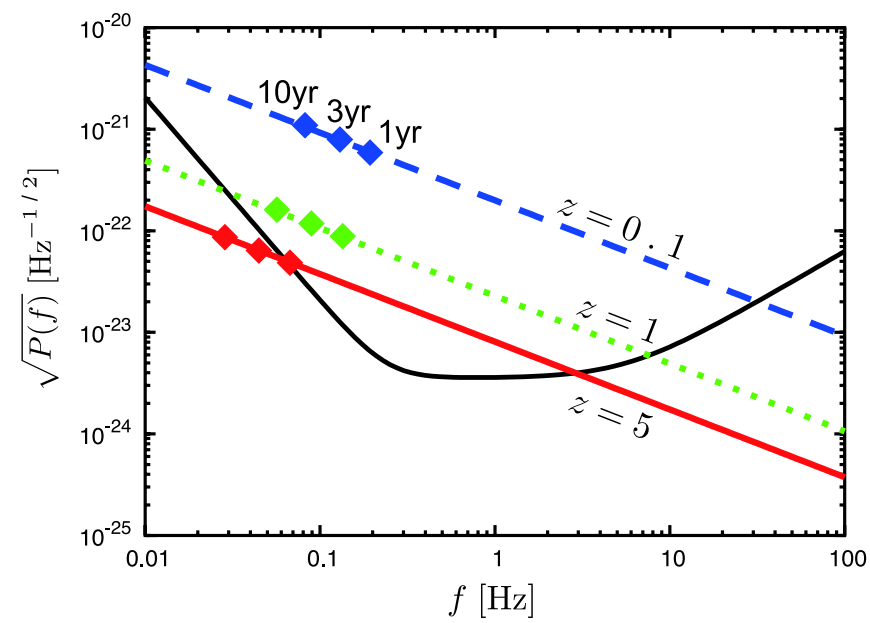

FIG. 2 (color online). Sky-averaged DECIGO noise curve. (Arm angle $60^{\circ}$ is taken into account.) Diagonal lines represent frequency evolutions of an NS-NS binary at $z=5$ (solid red line), $z=1$ (dotted green line), and $z=0.1$ (dashed blue line). Diamonds on the lines from the right to the left denote the frequency of the binary $1 \mathrm{yr}, 3 \mathrm{yr}$, and $10 \mathrm{yr}$ before the merger. 


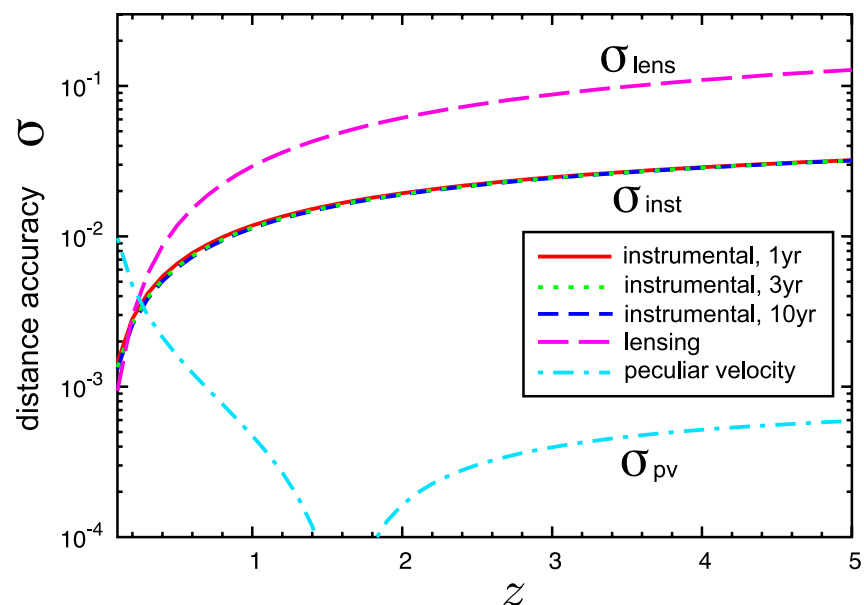

FIG. 3 (color online). Measurement accuracy of the luminosity distance with a single binary as a function of redshifts. The curves tagged $\sigma_{\text {inst }}$ are those determined only by instrumental noise and with the observation time $1 \mathrm{yr}$ (solid red curve), $3 \mathrm{yr}$ (dotted green curve), and $10 \mathrm{yr}$ (short-dashed blue curve), respectively. The lensing error and the peculiar-velocity error are represented by magenta (long-dashed) and light blue (dashdotted) curves.

$$
\Delta \theta_{a}=\sqrt{\left\{\Gamma^{-1}\right\}_{a a}}
$$

In Fig. 3, the resultant error of the luminosity distance for a single binary, $\sigma_{\text {inst }}$, is plotted against a source redshift, assuming the observation time $1 \mathrm{yr}$ (solid curve), $3 \mathrm{yr}$ (dotted curve), and $10 \mathrm{yr}$ (dashed curve). The overlap of these three curves indicates that $\sigma_{\text {inst }}$ hardly depends on the observation time, because the observation time appears only through the cutoff frequency $f_{\text {min }}$ with the fractional power of $3 / 8$ and the improvement of the precision is generally slow. Even for a single binary system, the precision of a few percent levels is easily achievable for the distance measurement in the absence of systematic errors, and this is also true for a rather high- $z$ binary.

\section{B. Accuracy of Hubble parameter}

Given the uncertainty of the averaged luminosity distance for each binary, the accuracy of the Hubble parameter is estimated from Eq. (11), and with the ensemble over the $\Delta N(z)$ independent binary systems in the vicinity of the redshift $z$, we can get an improved constraint on the Hubble parameter at each redshift bin.

Here, to derive the measurement error of the Hubble parameter, we adopt the following fitting form of the NSNS merger rate given by Ref. [26]:

$$
\dot{n}(z)=\dot{n}_{0} r(z) ; \quad r(z)= \begin{cases}1+2 z & (z \leq 1) \\ \frac{3}{4}(5-z) & (1<z \leq 5), \\ 0 & (5<z)\end{cases}
$$

where the function $r(z)$ is estimated based on the star formation history inferred from the UV luminosity [27]. The quantity $\dot{n}_{0}$ represents the merger rate at present. Though it is still uncertain, we adopt the most recent estimate, $\dot{n}_{0}=10^{-6} \mathrm{Mpc}^{-3} \mathrm{yr}^{-1}$, as a reliable and confident estimate based on extrapolations from the observed binary pulsars in our Galaxy [28]. Then, the number of NS binaries in the redshift interval $[z-\Delta z / 2, z+\Delta z / 2]$ observed during $T_{\mathrm{obs}}, \Delta N(z)$, is given by [26]

$$
\Delta N(z)=T_{\mathrm{obs}} \int_{z-\Delta z / 2}^{z+\Delta z / 2} d V_{c}\left(z^{\prime}\right) \frac{\dot{n}\left(z^{\prime}\right)}{1+z^{\prime}}
$$

where $d V_{c}$ means the comoving volume element defined as $d V_{c}(z)=4 \pi r^{2}(z) d z / H(z)$ with the comoving radial distance $r(z)=d_{L}(z) /(1+z)$.

In Fig. 4, observed redshift distribution of NS binaries $\Delta N(z)$ is plotted, assuming the $3 \mathrm{yr}$ observation and the redshift width $\Delta z=0.1$. The total number of NS binaries is $\sim 10^{6}$, which is much larger than the expected number of type Ia supernovae. Note that the number of merger events increases with $T_{\text {obs }}$, and thus the accuracy of the Hubble parameter is improved by a factor $T_{\mathrm{obs}}^{1 / 2}$. Combining this and the distance error in previous subsection, Fig. 5 shows the expected errors for the Hubble parameter measured from the dipole anisotropy. The three different error bars in each redshift bin represent the results from the 1-, 3-, and 10-yr observations (from large to small sizes). The figure implies that up to the redshift $z=1$, the Hubble parameter can be accurately measured with a precision of $2-5 \%, 1-3 \%$, and $0.7-1.5 \%$ for the observation time of 1,3 , and $10 \mathrm{yr}$, respectively. Even at $z=2$, the Hubble parameter can be measured with a precision of $18 \%, 10 \%$, and $6 \%$ for the

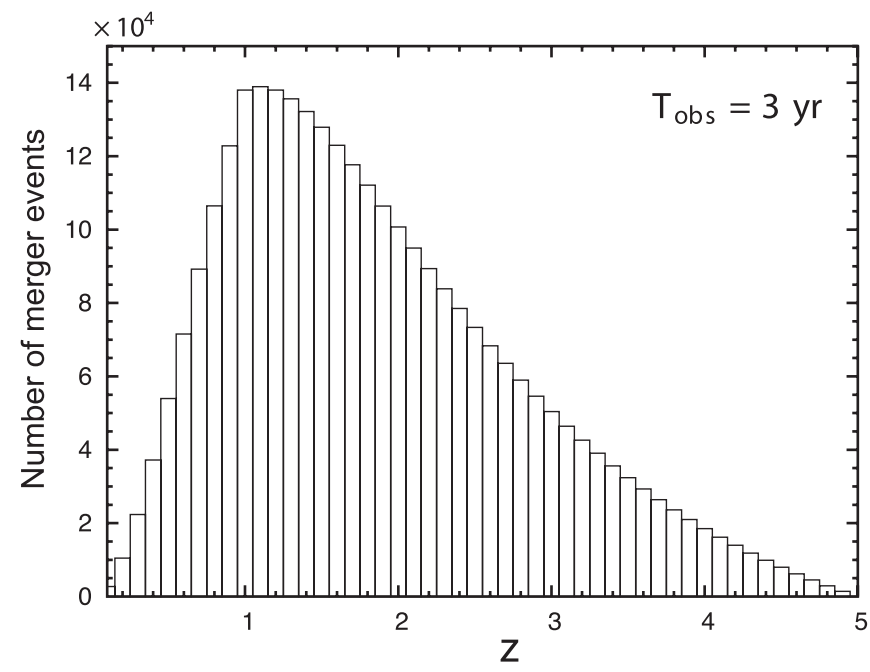

FIG. 4. Number of NS-NS binaries (in the unit of $10^{4}$ ) that would be observed by DECIGO in each redshift bin of $\Delta z=0.1$ at a redshift $z$ during $3 \mathrm{yr}$ observation. As is manifest from Eq. (18), the number of the binaries scales linearly with $T_{\text {obs }}$. 


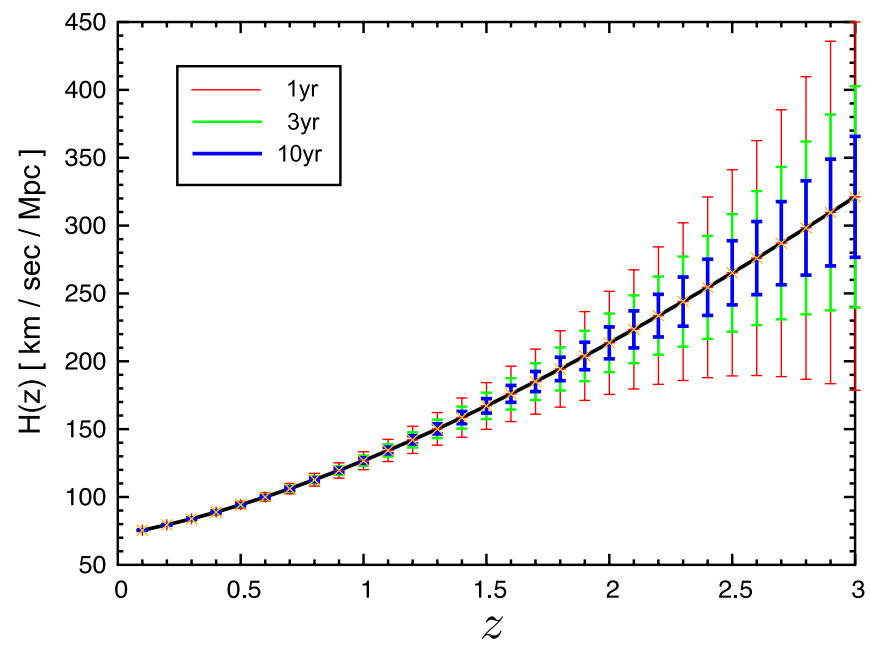

FIG. 5 (color online). The Hubble parameter calculated with our fiducial cosmological parameters (solid curve) and $1 \sigma$-error bars estimated in the cases that we use all binaries observed by DECIGO during the observation time, $1 \mathrm{yr}$ (red), $3 \mathrm{yr}$ (green), and $10 \mathrm{yr}$ (blue). Long observation time corresponds to the smaller error bar.

observation time of 1,3 , and $10 \mathrm{yr}$, respectively. This is quite impressive in the sense that a GW standard siren has a nearly equal sensitivity to the Hubble parameter with other complementary methods such as BAO. Another noticeable point using the standard sirens is that we can trace the redshift evolution of Hubble parameter even at higher redshift $z \gtrsim 1$. Although the number of high- $z$ NS binaries is highly uncertain, the standard sirens would be potentially powerful to probe the early-time cosmic expansion, and deserves further investigation.

\section{SYSTEMATIC ERRORS}

So far, we have discussed the accuracy of the Hubble parameter taking only an account of the distance error associated with the instrumental noise. However, there are several effects which may systematically affect the measurement of dipole anisotropies in the luminosity distance, leading to increasing the error in the Hubble parameter. Among them, a dominant contribution may come from the gravitational lensing magnification induced by the matter inhomogeneities of large-scale structure along the line of sight (e.g., [29-32]), which systematically changes the luminosity distance to each binary system. Another important effect would be the peculiar velocity of the binary along the line of sight, which randomly contributes to measurement error via Doppler effect. These systematic errors to the averaged luminosity distance are summarized as

$$
\left[\frac{\Delta d_{L}^{(0)}(z)}{d_{L}^{(0)}(z)}\right]^{2}=\sigma_{\text {inst }}^{2}(z)+\sigma_{\text {lens }}^{2}(z)+\sigma_{\mathrm{pv}}^{2}(z)
$$

where $\sigma_{\text {inst }}$ is the error associated with the GW experiment in Sec. III A, $\sigma_{\text {lens }}$ is the lensing error, and $\sigma_{\mathrm{pv}}$ is the peculiar-velocity error.

There are several studies on the effect of lensing magnification, particularly focusing on the distance measurement from the type Ia supernovae. Holz and Linder [30] estimated the lensing error on the distance measurement by using Monte Carlo simulation, and, assuming the Gaussian form of lensing magnification probability, they derived a fitting formula for the systematic error. Later, the significance of the non-Gaussian tail has been recognized $[33,34]$, and it turns out that this effect reduces the lensing error by a factor of 1.5-2, compared to the Gaussian distribution. More recently, Hirata, Holz, and Cutler [33] adopted a log-normal distribution for the magnification probability and obtained the fitting formula for the (averaged) distance error:

$$
\sigma_{\text {lens }}(z)=0.066\left[\frac{1-(1+z)^{-0.25}}{0.25}\right]^{1.8} .
$$

In what follows, we adopt the lensing error in Eq. (20).

As for the peculiar-velocity error, the clustering of galaxies induced by the gravity leads to a coherent and/or virialized random motion, which gives rise to the Doppler effect and affects the determination of cosmological redshift via the spectroscopic measurement. In addition, binary barycentric motion itself in the host galaxy also leads to the Doppler effect, which causes random fluctuations in the luminosity distance. These two systematic effects can be of the same order and can be translated into the distance error as [35]

$$
\sigma_{\mathrm{pv}}(z)=\left|1-\frac{(1+z)^{2}}{H(z) d_{L}(z)}\right| \sigma_{\mathrm{v}, \mathrm{gal}} .
$$

Here, $\sigma_{\mathrm{v}, \mathrm{gal}}$ is the 1-dimensional velocity dispersion of the galaxy. Taking account of the nonlinear effect of gravity, it often set to $\sigma_{\mathrm{v}, \mathrm{gal}}=300 \mathrm{~km} \mathrm{~s}^{-1}$, mostly independent of the redshifts [36].

In Fig. 3, the distance errors for a single binary system from the lensing magnification, $\sigma_{\text {lens }}$, and peculiar velocity, $\sigma_{\mathrm{pv}}$, are overlaid, together with the uncertainty from the GW observations. It turns out that the lensing magnification could dominate the distance error, and exceeds the error from the $\mathrm{GW}$ experiments at $z \gtrsim 0.2$. Thus, the lensing magnification could be potentially a main source of the distance error, and the accuracy of the Hubble parameter might be somewhat degraded.

In Fig. 6, the size of the measurement error for the Hubble parameter, $\Delta H(z) / H(z)$, is plotted as function of redshift, taking account of all the systematics and the binary distribution. Compared with the case without lensing error (dotted curves), the resultant accuracy of Hubble parameter are degraded. Nevertheless, even including the lensing systematics, the Hubble parameter up to $z \lesssim 1$ can 
TRACING THE REDSHIFT EVOLUTION OF HUBBLE ...

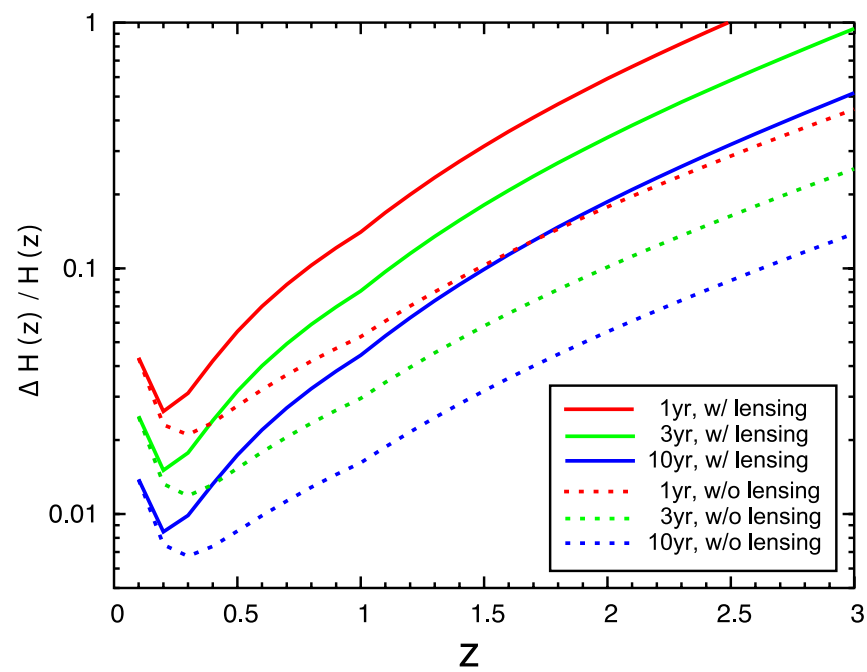

FIG. 6 (color online). Measurement accuracy of the Hubble parameter with all observed binaries. We plot the measurement accuracies, including the lensing error, with the observation time $T_{\text {obs }}=1 \mathrm{yr}, 3 \mathrm{yr}$, and $10 \mathrm{yr}$ from the top to the bottom, respectively (solid curves), and those without the lensing error (dotted curves).

be accurately measured with the precision $3-14 \%, 1.5-8 \%$, and $0.8-4 \%$, for $T_{\mathrm{obs}}=1 \mathrm{yr}, 3 \mathrm{yr}$, and $10 \mathrm{yr}$, respectively. Although the lensing effect is potentially crucial for the cosmological application of standard sirens, the technique to reduce the lensing effect has been recently exploited $[33,34,37,38]$, and the feasibility of the method has been discussed. With an improved technique developed in the near future, the lensing systematics would be removed, and one could approach the limit determined by the instrumental noise.

We briefly comment on the uncertainty in the amplitude of dipole $\mathbf{v}_{0}$. The current constraint on the motion of the local observer comes from the CMB observation, and the estimated error of $\mathbf{v}_{0}$ directly affects the accuracy of Hubble parameter in Eq. (11). However, the current observation produces

$$
\frac{\Delta H(z)}{H(z)}=\frac{\Delta\left|\mathbf{v}_{0}\right|}{\left|\mathbf{v}_{0}\right|} \approx 2.44 \times 10^{-3} .
$$

Therefore, the systematics in the dipole from the CMB observation give a tiny contribution, and can be ignored.

Finally, note that the results obtained here rely on the fact that we can successfully identify the redshifts of all host galaxies. In practice, the identification would be as difficult as increasing the redshift, because galaxies at high $z$ are fainter and the time required for a spectroscopic
PHYSICAL REVIEW D 83, 084045 (2011)

measurement is much longer than that for low- $z$ galaxies. The resultant accuracy of the Hubble parameter measurement would be degraded, being proportional to $1 / \sqrt{N(z)}$, where $N(z)$ is the number of galaxies in a bin at the redshift $z$. Thus, the measurement accuracy depends on the capability of galaxy redshift survey available in the future.

Another important issue is detector calibration, which would potentially affect the measurement accuracy of the luminosity distance. It is rather crucial not only for the Hubble parameter measurment, but also for the subtraction of the neutron star binary foreground in order to achieve the detection of an inflationary gravitational-wave background as a primary science goal of DECIGO/BBO. Although this is beyond the scope of this paper, the issue should be considered seriously and addressed in the future.

\section{SUMMARY}

In this paper, we have shown that the redshift evolution of Hubble parameter can be measured by utilizing a large number of NS binaries observed by space-based GW detectors such as DECIGO and BBO. Although this method requires a large number of samples $10^{5}-10^{6}$ to measure $H(z)$ with the accuracy of a few \%, DECIGO and BBO will detect enough numbers of samples up to high redshifts. Including the lensing magnification, the Hubble parameter as a function of redshift up to $z=1$ can be determined with the accuracy of $3-14 \%, 1.5-8 \%$, and $0.8-4 \%$, for the observation time $T_{\mathrm{obs}}=1,3$, and $10 \mathrm{yr}$, respectively. With a technique to remove the lensing magnification, we can further improve the accuracy by a factor of $\sim 2.7$ at $z=1$. Although the feasibility of the method, particularly on the redshift identification of the host galaxies and the detector calibration, still needs to be investigated in more detail, the present method puts forward an interesting possibility to enlarge the science with standard sirens as a by-product, and it is complementary to other methodologies to measure the Hubble parameter.

\section{ACKNOWLEDGMENTS}

We acknowledge N. Kanda, T. Tanaka, K. Yagi, J. Yokoyama, and C. Yoo for helpful discussions and valuable comments. We also thank T. Smith for his effort to improve our manuscript. A. N. and S. S. are supported by a Grant-in-Aid through JSPS. S. S. is also supported by JSPS through Excellent Young Researchers Overseas Visit Program. A. T. and S. S. are supported in part by Grantsin-Aid for Scientific Research from the JSPS (No. 21740168 for A. T. and No. 21-00784 for S. S.). 
[1] N. Seto, S. Kawamura, and T. Nakamura, Phys. Rev. Lett. 87, 221103 (2001).

[2] S. Sato et al., J. Phys. Conf. Ser. 154, 012040 (2009).

[3] E.S. Phinney et al., "Big Bang Observer, NASA Mission Concept Study,” NASA 2003 (unpublished).

[4] C. Cutler and D. E. Holz, Phys. Rev. D 80, 104009 (2009).

[5] K. Yagi and T. Tanaka, Prog. Theor. Phys. 123, 1069 (2010).

[6] A. Nishizawa, A. Taruya, and S. Kawamura, Phys. Rev. D 81, 104043 (2010).

[7] B. F. Schutz, Nature (London) 323, 310 (1986).

[8] Strictly speaking, this is true for flat geometry of the Universe. For closed and open universe, $H(z)$ is replaced with $H(z) \cos ^{-1}\left[\int_{0}^{z} \frac{d z^{\prime}}{H\left(z^{\prime}\right)}\right], \quad H(z) \cosh ^{-1}\left[\int_{0}^{z} \frac{d z^{\prime}}{H\left(z^{\prime}\right)}\right], \quad$ respectively.

[9] C. Bonvin, R. Durrer, and M. Kunz, Phys. Rev. Lett. 96, 191302 (2006).

[10] C. Bonvin, R. Durrer, and A. Gasparini, Phys. Rev. D 73, 023523 (2006).

[11] D. Stern, R. Jimenez, L. Verde, M. Kamionkowski, and S. A. Stanford, J. Cosmol. Astropart. Phys. 02 (2010) 008.

[12] E. Gaztanaga, R. Miquel, and E. Sanchez, Phys. Rev. Lett. 103, 091302 (2009).

[13] E. Gaztanaga, A. Cabre, and L. Hui, Mon. Not. R. Astron. Soc. 399, 1663 (2009).

[14] M. Sasaki, Mon. Not. R. Astron. Soc. 228, 653 (1987).

[15] Nevertheless, the gravitational lensing magnification might be statistically measurable and has been recently discussed in Ref. [4].

[16] N. Jarosik et al., Astrophys. J. Suppl. Ser. 192, 14 (2011).

[17] P. S. Corasaniti, M. L. Verde, A. Crotts, and C. Blake, Mon. Not. R. Astron. Soc. 369, 798 (2006).

[18] A. Crotts et al., arXiv:astro-ph/0507043.

[19] C. Cutler and E. E. Flanagan, Phys. Rev. D 49, 2658 (1994).

[20] M. Maggiore, Gravitational Waves (Oxford University Press, New York, 2008).

[21] For the geometric factor arising from the nonorthogonal detector arms $\left(60^{\circ}\right)$, we incorporate this effect into the detector noise curve $P(f)$. The factor arising from detector's angular response is also taken into account in evaluating the noise curve by averaging over the sky.

[22] R. Takahashi and T. Nakamura, Prog. Theor. Phys. 113, 63 (2005).

[23] L. S. Finn, Phys. Rev. D 46, 5236 (1992).

[24] T. A. Prince, M. Tinto, S. L. Larson, and J. W. Armstrong, Phys. Rev. D 66, 122002 (2002).

[25] The noise power spectrum presented here is slightly different from the one given by Ref. [6], who considered orthogonal detector arms and optimal incidence of a GW.

[26] C. Cutler and J. Harms, Phys. Rev. D 73, 042001 (2006).

[27] R. Schneider, V. Ferrari, S. Matarrese, and S. F. P. Zwart, Mon. Not. R. Astron. Soc. 324, 797 (2001).

[28] J. Abadie et al., Classical Quantum Gravity 27, 173001 (2010).

[29] J. Wambsganss, R. Cen, G. Xu, and J.P. Ostriker, Astrophys. J. 475, L81 (1997).

[30] D.E. Holz and E. V. Linder, Astrophys. J. 631, 678 (2005).

[31] D.E. Holz and R. M. Wald, Phys. Rev. D 58, 063501 (1998).

[32] K. Kainulainen and V. Marra, Phys. Rev. D 83, 023009 (2011).

[33] C. M. Hirata, D. E. Holz, and C. Cutler, Phys. Rev. D 81, 124046 (2010).

[34] C. Shang and Z. Haiman, Mon. Not. R. Astron. Soc. 411, 9 (2011).

[35] C. Gordon, K. Land, and A. Slosar, Phys. Rev. Lett. 99, 081301 (2007).

[36] L. Silberman, A. Dekel, A. Eldar, and I. Zehavi, Astrophys. J. 557, 102 (2001).

[37] C. Shapiro, D. J. Bacon, M. Hendry, and B. Hoyle, Mon. Not. R. Astron. Soc. 404, 858 (2010).

[38] S. Hilbert, J. R. Gair, and L. J. King, Mon. Not. R. Astron. Soc. 412, 1023 (2011). 